\title{
ESTIMATION OF THE MODULATION INDEX OF CPM SIGNALS USING HIGHER-ORDER STATISTICS
}

\author{
Javier R. Fonollosal and José A. R. Fonollosa ${ }^{2}$ \\ ${ }^{1}$ Signal and Image Processing Institute, University of Southern California, 3740 McClintock Ave Room 400, \\ Los Angeles CA 90089-2564, USA \\ 2Universitat Politècnica de Catalunya. E.T.S.E. Telecomunicació, Apdo 30.002, Barcelona 08080, Spain
}

\begin{abstract}
Three simple methods are proposed for the estimation of the modulation index of Continuous Phase Modulated signals in noise. These methods employ the estimated autocorrelation and fourth-order cumulant sequences of the received signal after sampling at the symbol rate. Analytic expressions are derived for the asymptotic mean and variance of the estimated parameters which are corroborated by means of Monte Carlo simulations. The performance of the methods is illustrated graphically and numerically. It is concluded that, under significant noise degradation, only the scheme based on the fourth-order cumulant sequence can be used to consistently estimate the modulation index $h$ in the range $0<h<1$.
\end{abstract}

\section{INTRODUCTION AND PROBLEM FORMULATION}

Continuous Phase Modulations (CPM) represent an important class of communication signals due to their bandwidth efficiency. One of the most important parameters that define CPM signals is the modulation index. In this paper, we study the estimation of this parameter for demodulation purposes.

CPM digital communication signals are given by [1],

$$
\begin{gathered}
s(t)=A \cos \left[2 \pi f_{c} t+\phi(t ; I)+\phi_{0}\right] \\
\phi(t ; I)=2 \pi h \sum_{k=-\infty}^{\infty} I_{k} q\left(t-k T_{s}\right),
\end{gathered}
$$

where $A$ is the amplitude, $f_{C}$ is the carrier frequency, $\phi_{0}$ is a constant phase, $h$ is the modulation index and $I_{k}$ are the transmitted symbols. Each symbol in the sequence can take one out of $M$ values $\{ \pm 1, \pm 3, \ldots, \pm(M-1)\}$. These symbols are assumed statistically independent and identically distributed. The waveform $q(t)$ may be represented in general as the integral of some pulse $u(t)$. If $u(t)=0$ for $t>T_{s}$, where $T_{S}$ is the symbol period, the CPM signal is called full response CPM. If $u(t) \neq 0$ for $t>T_{S}$, the modulated signal is then called partial response CPM. In the sequel, we will consider only full response CPM signals.

To estimate the modulation index $h$, the equivalent lowpass signal $v(t)$ is sampled to derive the discrete sequence $v(n)$. The sampling period is denoted by $T_{o}$.

$$
\begin{gathered}
v(t)=A \exp (j \phi(t ; I)) \\
v(n)=A \exp \left[j 2 \pi h \sum_{k=-\infty}^{n T_{o} / T_{s}} I_{k} q\left(n T_{o^{-}} k T_{s}\right)\right]
\end{gathered}
$$

\section{AUTOCORRELATION AND FOURTH- ORDER CUMULANT SEQUENCES}

The autocorrelation and power spectrum of CPM signals have been studied thoroughly in the literature (see for example [2]). For binary CPM signals with $h<1$ and sampling at the symbol rate, i.e. $T_{s}=T_{o}, v(n)$ is stationary and for its autocorrelation function $a_{v}(m)$ we obtain,

$$
a_{v}(m)=A^{2} \cos (\pi h)^{/ m /}
$$

The modulation index $h$ can be obtained from the autocorrelation of the received sequence. However, if the CPM signal is observed in noise, the autocorrelation of the noise is added to the autocorrelation of the signal making the estimation of $h$ biased. Fig. 1 shows the autocorrelation function $a_{v}(m)$ as a function of the modulation index. 


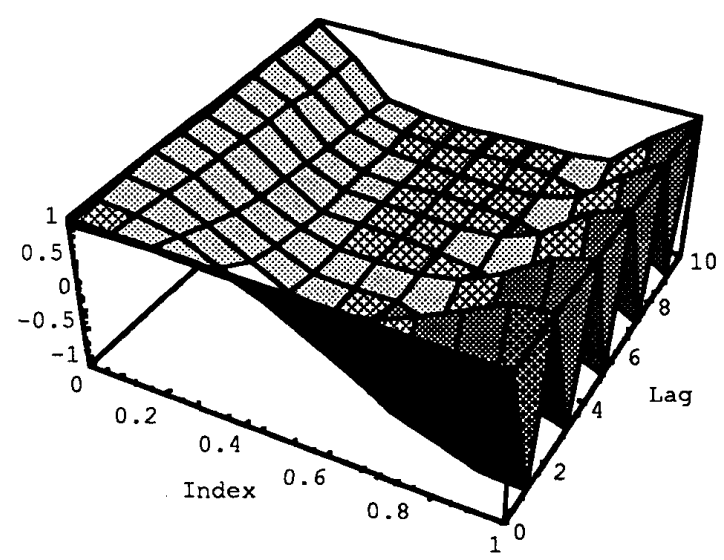

Figure 1: Autocorrelation as a function of the modulation index.

We have studied the higher-order moments and cumulants of CPM signals. They can provide us with consistent unbiased estimates of the modulation index $h$ even when the received sequence is corrupted by additive Gaussian noise of unknown covariance. In particular, sampling at the symbol rate, i.e. $T_{s}=T_{o}$, a slice of the fourth-order cumulant of $v(n)$ with $h<1$ admits the following expression,

$$
c_{v}(0,0, m)=-A^{4} \cos (\pi h)^{/ m /}
$$

which can also be used to estimate $h$. Fig. 2 shows the cumulant sequence $c_{v}(0,0, m)$ as a function of the modulation index.

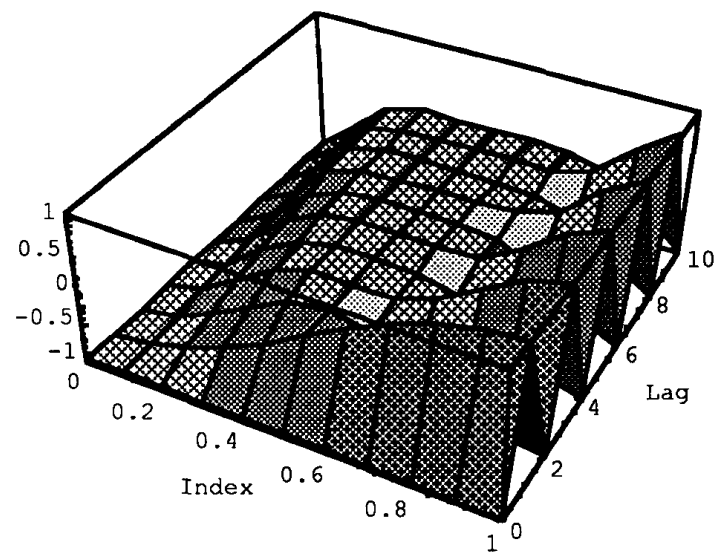

Figure 2: Fourth-order cumulant as a function of the modulation index.

\section{ESTIMATION OF THE MODULATION INDEX.}

We denote by $\hat{a}(m)$ and $\hat{c}(0,0, m)$ the real part of the estimated autocorrelation and fourth-order cumulant of the received signal after sampling at the symbol rate. We analyzed three simple methods that can be used to estimate $\cos [\pi h]$. Two of them employ the estimated autocorrelation whereas the third one makes use of the fourth-order cumulant sequence. They are defined as,

$$
\begin{gathered}
c_{1}=\hat{a}(1) / \hat{a}(0) \\
c_{2}=\hat{a}(2) / \hat{a}(1) \\
c_{3}=\hat{c}(0,0,1) / \hat{c}(0,0,0)
\end{gathered}
$$

Since $c_{l}$ employs the power of the received sequence, it is clearly sensitive to the SNR. The estimation given by $c_{2}$ is asymptotically unaffected by white noise but can not be used for values of $h$ near 0.5 when $a(1)$ tends to zero. In addition, $c_{2}$ is biased if the noise is colored. On the other hand, $c_{3}$ provides asymptotically unbiased estimates in white or colored Gaussian noise.

We derived analytic expressions for the bias and variance of $c_{1}, c_{2}$, and $c_{3}$ as a function of the modulation index and the SNR. This analytic performance analysis has been confirmed by means of Monte Carlo simulations.

\section{ANALYTIC PERFORMANCE ANALYSIS}

We study here the asymptotic mean and variance of the three estimation methods proposed above. These results hold exactly only in the limit as the number of samples $N$, approaches infinity. Nevertheless, they are sufficiently accurate for values of $N$ in the range of interest.

Analytic performance evaluation of algorithms based on sample cumulants requires computation of the covariances of these statistics, and the Jacobian matrix of the estimates with respect to them [4].

In the three proposed methods the estimates are obtained as the quotient of two sample statistics:

$$
e=\frac{\hat{s}_{1}}{\hat{s}_{2}}
$$

In this case, the asymptotic mean and variance of the estimate $e$ is given by: 


$$
\begin{gathered}
E\{e\}=\frac{E\left\{\hat{s}_{1}\right\}}{E\left\{\hat{s_{2}}\right\}}=\frac{s_{1}}{s_{2}} \\
\operatorname{var}\{e\}=\frac{s_{1}^{2}}{s_{2}{ }^{2}}\left(\frac{v_{11}}{s_{1}{ }^{2}}-2 \frac{v_{12}}{s_{1} s_{2}}+\frac{v_{22}}{s_{2}{ }^{2}}\right)
\end{gathered}
$$

where $v_{11}=\operatorname{var}\left\{s_{1}\right\}, v_{22}=\operatorname{var}\left\{s_{2}\right\}$ and $v_{12}=\operatorname{cov}\left\{s_{1}, s_{2}\right\}$ are the asymptotic covariances of the sample statistics $s_{1}$ and $s_{2}$.

Hence, we need to compute the asymptotic covariances of the statistics considered in (5) to obtain the variances of the estimates. Following a similar procedure to the one described in [4], we derived general expressions for the covariances of sample cumulants of CPM signals in noise from the covariances of sample moments.

Hand computation of the covariances of sample fourthorder moments is very tedious since a summation of eighth-order moments of the communication signal in noise is necessary. A symbolic mathematical package [3], has demonstrated to be very useful in this task.

Substitution of the resulting covariances in (8) gives the performance of the three estimation methods. Only the final results are given.

Let us define

$$
\begin{aligned}
& r=\sigma_{n}^{2} / A^{2} \\
& a=\cos [2 \pi h]
\end{aligned}
$$

where $A$ is the amplitude of the CPM signal and $\sigma_{n}{ }^{2}$ is the variance of the white Gaussian noise. The asymptotic mean and variance of the estimates $c_{1}, c_{2}$, and $c_{3}$ are:

$$
\begin{gathered}
E\left\{c_{1}\right\}=\frac{\cos [\pi h]}{(1+r)} \\
\operatorname{var}\left\{c_{1}\right\}=\frac{(1-a) r+(4-a) r^{2}+(5+a) r^{3}+r^{4}}{2(1+r)^{4} N} \\
\operatorname{var}\left\{c_{2}\right\}=\frac{2-4 a+2 a^{2}+(4-4 a) r+(6+2 a) r^{2}}{\left.8 c_{2}\right\}=\cos [\pi h]} \\
\operatorname{var}\left\{c_{3}\right\}=\frac{(1-a) r+3 r^{2}+(16+8 a) r^{3}+(6+4 a) r^{A}}{2 N}
\end{gathered}
$$

\section{EXAMPLE}

The following figures illustrate the performance of the proposed methods for the estimation of the modulation index of a binary full response CPM signal in white Gaussian noise for $S N R=0 d B$, and a sequence length of $N=1000$ samples.

Fig. 3 shows the asymptotic performance of the first estimation method $c_{1}$. The dotted line indicates the mean of the estimate while the dashed lines the mean $+/$ - the standard deviation. The solid line is the real value of $\cos (\pi h)$. As we mentioned before the method is clearly biased in the presence of noise.

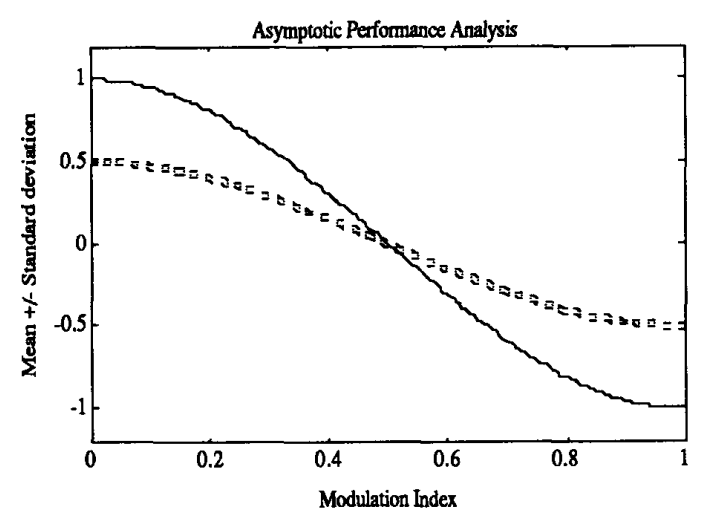

Figure 3: Performance analysis of $c_{1}$.

The performance of the estimation obtained with $c_{2}$ is given in Fig. 4. In this case the method is not biased and the real value of $\cos (\pi h)$, solid line, coincides with the mean of the estimation. The mean $+/-$ the standard deviation are indicated by the dashed lines. We can observe how the estimation degrades as we approach the critical value of $h=0.5$ where the variance goes to infinity.

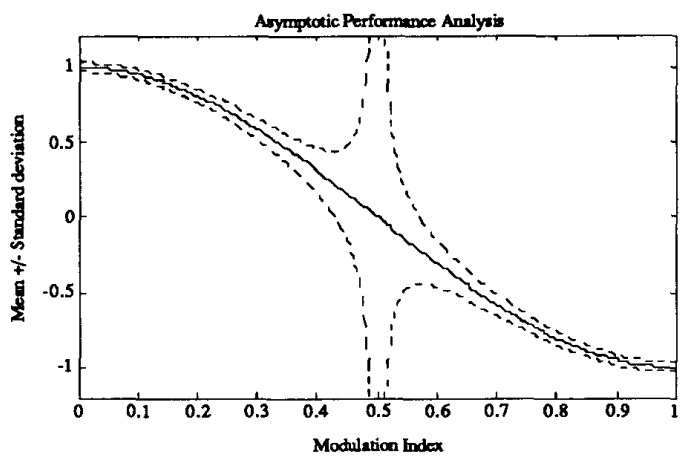

Figure 4: Performance analysis of $c_{2}$ 


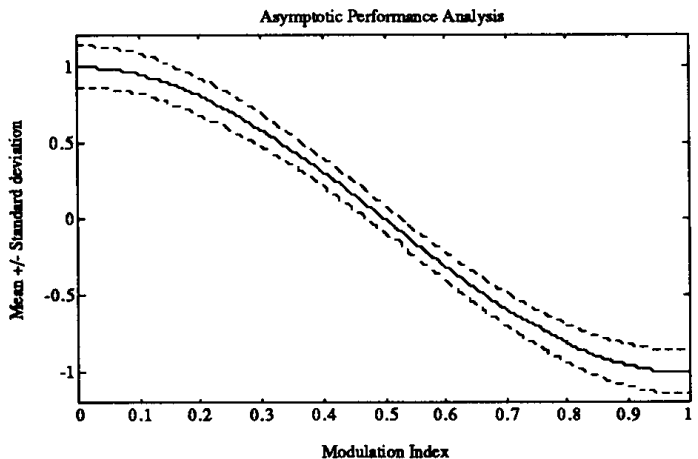

Figure 5: Performance analysis of $c_{3}$

Fig. 5 illustrates the performance of $c_{3}$. The method is again unbiased and thus, the real value of $\cos (\pi h)$, solid line, coincides with the mean of the estimation. The mean $+/$ - the standard deviation are indicated by the dashed lines. A relatively small variance is attained independently of the value of $h$ in the range $0<h<1$.

The predicted analytic results were compared against simulation results for two values of the modulation index $h$. The mean and standard deviation of $c_{1}, c_{2}$, and $c_{3}$ is presented in Tables 1 and 2 . The first and second columns correspond to the simulation results while the third and fourth column give the analytic results.

The estimated autocorrelation and fourth-order cumulant sequences where computed from $N=1000$ samples of the received signal. The simulation results were obtained from a total of 100 Monte Carlo runs.

\begin{tabular}{|c|c|c|c|c|}
\cline { 2 - 5 } & $\begin{array}{c}\text { MonteCarlo } \\
\text { mean }\end{array}$ & $\begin{array}{c}\text { MonteCarlo } \\
\text { std }\end{array}$ & $\begin{array}{c}\text { Asymp. } \\
\text { mean }\end{array}$ & $\begin{array}{c}\text { Analytic } \\
\text { std }\end{array}$ \\
\hline$c_{1}$ & -0.4030 & 0.0181 & -0.4045 & 0.0183 \\
\hline$c_{2}$ & -0.8086 & 0.0423 & -0.8090 & 0.0444 \\
\hline$c_{3}$ & -0.8500 & 0.1466 & -0.8090 & 0.1212 \\
\hline
\end{tabular}

Table 1: Simulation versus analytic results for $h=0.8$, $\cos (\pi h)=-0.8090$.

\begin{tabular}{|c|c|c|c|c|}
\cline { 2 - 5 } \multicolumn{1}{c|}{} & $\begin{array}{c}\text { MonteCarlo } \\
\text { mean }\end{array}$ & $\begin{array}{c}\text { MonteCarlo } \\
\text { std }\end{array}$ & $\begin{array}{c}\text { Asymp. } \\
\text { mean }\end{array}$ & $\begin{array}{c}\text { Analytic } \\
\text { std }\end{array}$ \\
\hline$c_{1}$ & -0.0167 & 0.0180 & -0.0157 & 0.0196 \\
\hline$c_{2}$ & -0.3718 & 7.2121 & -0.0314 & 1.5910 \\
\hline$c_{3}$ & -0.0395 & 0.1002 & -0.0314 & 0.0867 \\
\hline
\end{tabular}

Table 2: Simulation versus analytic results for $h=0.51$, $\cos (\pi \mathrm{h})=-0.0314$.

\section{CONCLUSIONS}

In this paper we have addressed the problem of estimating the modulation index of Continuous Phase Modulated signals in noise. Three simple methods that employ the estimated autocorrelation and fourth-order cumulants have been proposed and analyzed.

We derived the analytic expressions of the covariances of sample cumulants of CPM signals in noise, and used these expressions to obtain the asymptotic mean and variance of the estimates. This analysis shows that, for low SNR, only the scheme based on the fourth-order cumulant sequence can be used to consistently estimate the modulation index $h$ in the range $0<h<1$. The Monte Carlo simulations confirm the predicted behavior for the three proposed methods.

\section{REFERENCES}

[1] Proakis, J.G., Digital Communications, McGrawHill, New York, NY, 1989.

[2] Anderson, J.B., Aulin, T. and Sundberg, C.W. Digital Phase Modulation Plenum, New York.

[3] Wolfram, S., Mathematica: A system for Doing Mathematics by Computer, Addison-Wesley, 1991.

[4] José A. R. Fonollosa, J. M. Mendel, "Analytic Performance Evaluation of Cumulant-Based FIR System Identification Methods", Proc. Sixth Signal Processing Workshop on Statistical Signal \& Array Processing. Victoria, BC, Canada 1992. 